\title{
Compact multi-wavelength filters in SOI using superimposed sidewall Bragg gratings
}

\author{
Michael J. Strain ${ }^{1,2}$, Stephen Thoms², Douglas S. MacIntyre'2, Marc Sorel ${ }^{2}$ \\ 1. Institute of Photonics, University of Strathclyde, Glasgow, G4 0NW, U.K. \\ 2. School of Engineering, University of Glasgow, Glasgow, G12 8LT, U. K.
}

Abstract - A compact silicon photonics multi-wavelength filter is demonstrated using superimposed sidewall Bragg gratings. We show arbitrary wavelength spacing with 8 superimposed gratings on a total footprint that is equivalent to a single Bragg grating device.

Integrated waveguide Bragg gratings are one of the fundamental building blocks in the Photonic Integrated Circuit (PIC) designer's toolkit. The concept of using the bandgap region of an inline grating device as a spectral filter is well established and has been used across material platforms for laser design [1], dispersion engineering [2] and sensing applications [3]. In the silicon-oninsulator (SOI) platform the high optical confinement of the mode to the grating area allows unprecedented control over the grating coupling coefficient and Bragg wavelength, producing a wide variety of filter designs in both amplitude and phase domains [4,5]. Furthermore the sinusoidal sidewall variation commonly employed to create the periodic grating function is easily implemented in standard nanofabrication lines and can be controllably engineered.

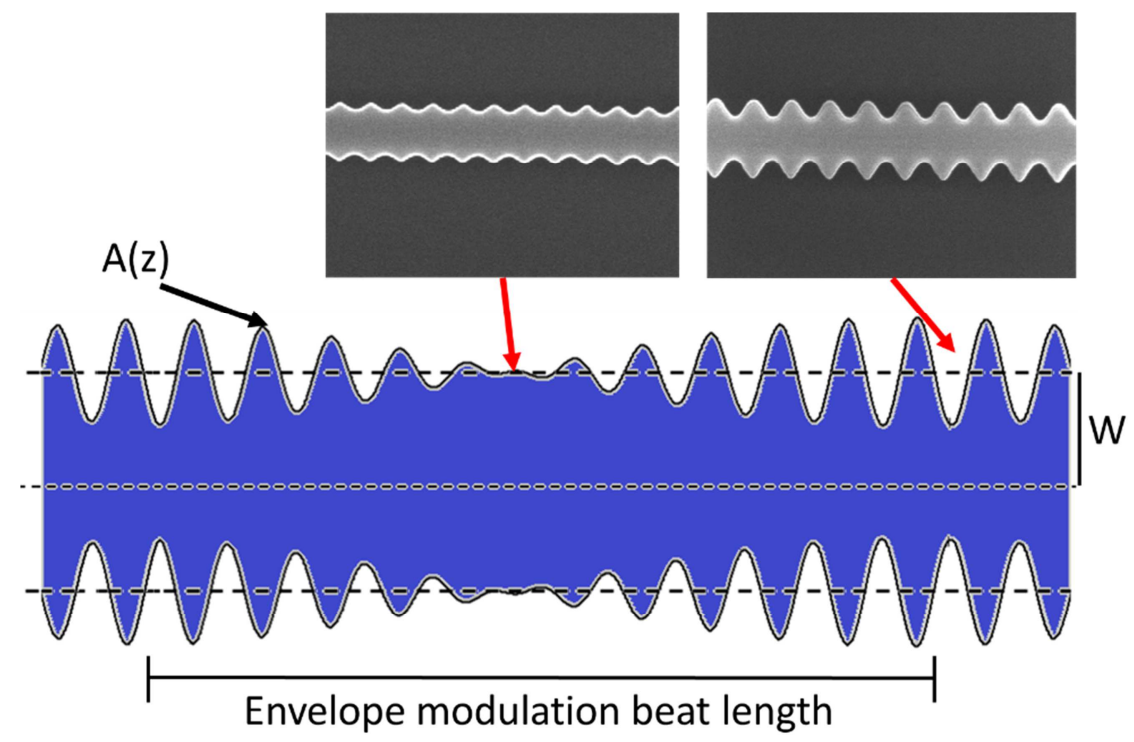

Fig. 1: Schematic of a superposition grating with two separate grating periods. SEM images show the amplitude variation of the sidewall grating along the device length due to beating between the two grating periods. The devices presented here are made by superimposing up to 8 grating periods over a total device length of $200 \mu \mathrm{m}$.

The simplest approach to achieving multiple filter bands in a single waveguide is by cascading individual gratings with variable Bragg wavelengths. This geometry, however, produces grating lengths of at least $N x L_{g}$, where $N$ is the number of required filter bands and $L_{g}$ is the individual grating length. In this work we demonstrate an alternative superstructured Bragg grating device that exhibits multiple filter bands with arbitrary wavelength spacings and with a footprint equivalent to a single Bragg grating device.

The superstructured grating design is similar in concept to the multi-exposure technique used in fibre grating designs. Single sidewall perturbation functions $A_{n}(z)$ can be defined as simple 
sinusoidal variations that produce the usual Bragg grating response, where $A_{n}(z)=a_{n} \sin \left(2 \pi z / \Lambda_{n}\right)$, and $\Lambda_{n}$ is the Bragg grating period for the $n^{\text {th }}$ filter response. The summation of these individual sidewall perturbation functions, $\sum_{n=1}^{N} A_{n}(z)$, produces a total sidewall perturbation function with $N$ Fourier components that will create $N$ grating responses within the same physical length as a classical single period grating. In addition, the extinction of the filters can be weighted using their individual perturbation amplitudes $a_{n}$. The device geometry is illustrated by the schematic of Fig. 1 that shows the profile of the sidewall grating when two separate grating periods are superimposed. In this work several grating devices based on an 8-wavelength basis set were fabricated and characterised. Arbitrary combinations of these 8 basis functions were shown to match simulated results and underline the equivalence of this device to a cascade of individual filters.

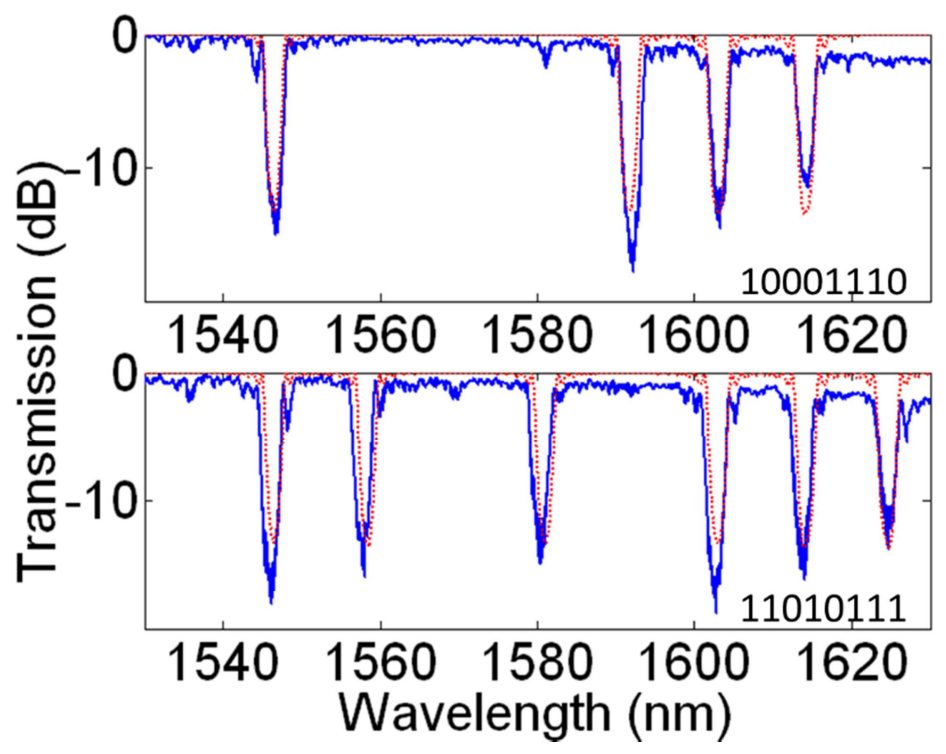

Fig. 2: Measured (blue curve) and simulated (red dotted curve) grating transmission spectra for two different superposition grating devices. The binary numbers indicate the gratings of the 8-basis wavelength set that were 'on' for each device.

The results of Fig. 2 indicate that any arbitrary combination of filter wavelengths can be fabricated in a single superposition with excellent agreement to the designed transfer function. Furthermore, the total length of the grating device is only $200 \mu \mathrm{m}$, which is far more compact than the equivalent serial grating geometry.

In conclusion we have demonstrated a compact means by which to generate a multiwavelength filter with non-repetitive filter band positions. Up to 8 grating filter bands are demonstrated in $200 \mu \mathrm{m}$ long devices on a single silicon waveguide. This same approach can be easily extended to the design of more complex transfer functions with arbitrary filter wavelengths that exhibit different reflectivities, bandwidth and phase profiles.

\section{References:}

[1] H. Ishii, K. Kasaya, and H. Oohashi, “Spectral Linewidth Reduction in Widely Wavelength Tunable DFB Laser Array,” IEEE J. Sel. Top. Quantum Electron., vol. 15, no. 3, pp. 514-520, 2009.

[2] M. J. Strain and M. Sorel, "Design and fabrication of integrated chirped Bragg gratings for on-chip dispersion control," Quantum Electron. IEEE J., vol. 46, no. 5, pp. 774-782, 2010.

[3] P. Prabhathan, V. M. Murukeshan, Z. Jing, and P. V Ramana, "Compact SOI nanowire refractive index sensor using phase shifted Bragg grating.," Opt. Express, vol. 17, no. 17, pp. 15330-41, Aug. 2009.

[4] J. T. Hastings, M. H. Lim, J. G. Goodberlet, and H. I. Smith, "Optical waveguides with apodized sidewall gratings via spatial-phaselocked electron-beam lithography," J. Vac. Sci. Technol. B Microelectron. Nanom. Struct., vol. 20, no. 6, p. $2753,2002$.

[5] G. F. R. Chen, T. Wang, C. Donnelly, and D. T. H. Tan, "Second and third order dispersion generation using nonlinearly chirped silicon waveguide gratings," Opt. Express, vol. 21, no. 24, p. 29223 , Nov. 2013. 